\title{
A checklist of Lecythidaceae in the upper Madeira River, Rondônia, Brazil with comments on diversity and conservation
}

\author{
Fernanda Silveira Catenacci ${ }^{1}$ and Marcelo Fragomeni Simon $^{2}$
}

${ }^{1}$ Programa de Pós-Graduação em Botânica, Departamento de Botânica, Instituto de Ciências Biológicas, Campus Universitário Darcy Ribeiro, Universidade de Brasília, Caixa Postal 4457, Brasília-DF, CEP: 70919-970, Brazil; e-mail: fer_catenacci@hotmail.com

${ }^{2}$ Embrapa Recursos Genéticos e Biotecnologia, Parque Estação Biológica (PqEB), Av. W5 Norte (final), Asa Norte, Caixa Postal 2372, Brasília-DF, CEP: 70770-917, Brazil; e-mail: marcelo.simon@embrapa.br

\begin{abstract}
The Lecythidaceae or Brazil Nut family are ubiquitous in non-flooded lowland forests of the Amazon, where they are indicative of well-preserved or little-disturbed habitats. A recent checklist of the Brazilian flora reported 10 genera and 119 species for that country, of which 104 are found in the Amazon region. However, the botanical knowledge in many regions of the country remains far from complete. This study aimed to analyze the diversity of Lecythidaceae in the upper Madeira River region in Rondônia, an area where botanical data is scarce. Lecythidaceae collections deposited in the CEN, INPA and RON herbaria were examined, and four field expeditions in the study area were carried out. For that region, we recorded 37 species of Lecythidaceae belonging to 7 genera: Eschweilera (21 species), Couratari (6), Cariniana (3), Gustavia (3), Allantoma (2), Bertholletia (1) and Lecythis (1). Among these species, five are listed as threatened in the IUCN Red List. The total number of species found in the upper Madeira River of Rondônia alone is higher than the 28 species reported to date for the entire state of Rondônia in the Brazilian Flora Checklist (Smith et al., 2015), and adds 13 new records of Lecythidaceae for the state. Considering that this study is restricted to the northern part of the state, our results suggest that the number of Lecythidaceae species in Rondônia is severely underestimated. Our findings highlight the need for more intensive floristic studies in the Amazon region, which should target areas that are botanically unexplored but have high biodiversity potential. Such studies will provide valuable information to support both taxonomic studies and species conservation assessments.
\end{abstract}

Keywords: Amazonia, conservation, field survey, flora, herbarium, Neotropics, new records.

Resumo. As Lecythidaceae, família da castanha-do-brasil, são ubíquas em florestas de terras baixas não inundáveis da Amazônia, onde são indicadoras de ambientes bem preservados ou pouco perturbados. Uma lista recente das espécies da flora do Brasil registrou 10 gêneros e 119 espécies para o país, das quais 104 são encontradas na região Amazônica. Porém, o conhecimento botânico em várias regiões do país permanece incompleto. Esse estudo tem como objetivo analisar a diversidade de Lecythidaceae na região do alto rio Madeira em Rondônia, uma área onde o conhecimento botânico é escasso. Coletas de Lecythidaceae depositadas nos herbários CEN, INPA e RON foram examinadas, e foram realizadas quatro expedições na área de estudo. Foram registradas para a região 37 espécies de Lecythidaceae pertencentes a 7 gêneros: Eschweilera (21 espécies), Couratari (6), Cariniana (3), Gustavia (3), Allantoma (2), Bertholletia (1) e Lecythis (1). Dentre essas espécies, cinco são listadas como ameaçadas na Lista Vermelha da IUCN. O número total de espécies encontradas apenas no alto rio Madeira em Rondônia é maior do que as 28 espécies registradas para todo o estado de Rondônia na Lista de Espécies da Flora do Brasil (Smith et al., 2015), e acrescenta 13 novos 
registros de Lecythidaceae para o estado. Considerando que esse estudo se restringe à parte norte do estado, nossos resultados sugerem que o número de espécies de Lecythidaceae para Rondônia encontra-se extremamente subestimado. Nossos resultados ressaltam a necessidade de realização de estudos florísticos intensivos na região Amazônica, os quais devem focar em áreas pouco exploradas botanicamente, mas com alto potencial para biodiversidade. Esses estudos poderão gerar informações relevantes para subsidiar tanto estudos taxonômicos quanto avaliação do estado de conservação de espécies.

The Lecythidaceae s.1., including Napoleonaeaceae and Scytopetalaceae, comprise a pantropical family of shrubs to large trees with about 350 species in 23 genera (Mori et al., 2007; Mori et al., 2010; Prance \& Kartawinata, 2013; Prance \& Jongkind, 2015). It is known worldwide as the Brazil Nut family because of the global trade of the seeds of Bertholletia excelsa Bonpl. The family is distributed in tropical regions of Africa, Madagascar, Mauritius, Asia, Australia, in the Pacific Islands and the Neotropics (Prance \& Mori, 1979; Mori et al., 2007).

The circumscription of the family is not well resolved, with a lack of consensus about the inter- and intrafamiliar relations of the group. Lecythidaceae s.s. as recently circumscribed (Mori et al., 2007; Mori et al., 2015; Huang et al., 2015) comprise three subfamilies: Barringtonioideae (erroneously referred as Planchonioideae in some publications), Foetidioideae and Lecythidoideae. Other studies include Napoleonaeoideae and Scytopetaloideae subfamilies in Lecythidaceae s.s. (APG II, 2003), but this broader circumscription would lead to taxonomic and nomenclatural inconsistencies (Mori et al., 2015). The relationships at lower taxonomic levels are also not completely resolved. Recent phylogenetic studies focused on Lecythidoideae, which includes the neotropical Lecythidaceae, support the monophyly of most genera (sensu Prance \& Mori, 1979; Mori \& Prance, 1990), but suggest that Eschweilera Mart. ex DC. and Lecythis Loefl. are not monophyletic and may be split into new genera (Huang et al., 2015; Mori et al., 2015).

Lecythidoideae, the subfamily with the largest number of species, is found from Mexico to Paraguay, occupying various types of habitats, but it is more diverse in the Guianas-Amazon floristic province, particularly in non-flooded lowland forests (Prance \& Mori, 1979). To date, Brazil has 10 genera and a total of 119 species of Lecythidaceae, of which 104 are found in the Amazon and 40 species are endemic to the country (Smith et al., 2015). Some species are very important economically in the Amazon: the edible seeds of Bertholletia excelsa are one of the principal products harvested in the biome, and several species of Allantoma Miers, Cariniana Casar. and Couratari Aubl., regionally referred as "tauari," are intensively exploited by the timber industry (Procópio et al., 2010). In the Amazon, the family can often be identified by a combination of vegetative characters: mostly arboreal habit, fibrous inner bark (locally called "envira"), a characteristic smell of linseed released when the bark is slashed, and simple and alternate leaves (Hopkins \& Mori, 1999). Neotropical Lecythidaceae are indicative of preserved or slightly disturbed lowland forests (Prance \& Mori, 1979) and comprise one of the most abundant families of the Amazon forests (ter Steege et al., 2013).

The aim of this study is to analyze the diversity of Lecythidaceae in the region of the upper Madeira River in the municipality of Porto Velho, Rondônia, Brazil. This region has suffered major environmental impacts over the last four decades due to agricultural expansion and infrastructure development. In the second volume of the Lecythidaceae monograph published in Flora Neotropica, Mori and Prance (1990) had already warned of the rapid process of deforestation in Rondônia and the existence of highly endangered species at that time. The construction of the BR364 highway in the 1980's expanded the state's agricultural frontier and led to the conversion of more than $75 \%$ of its forests into pastures (Ferraz et al., 2005). More recently, hydroelectric dams 
have been built in the Madeira River basin (Moser et al., 2014), representing another threat to natural habitats. Although the municipality of Porto Velho accounts for $60 \%$ of total Lecythidaceae records in Rondônia (according to the speciesLink network, www.splink.org.br), the area remains poorly explored botanically compared to relatively well-collected areas such as Manaus and Belém (Hopkins, 2007).

Considering this scenario of rampant deforestation and rapid habitat loss, it is crucial to increase the knowledge of the composition and distribution of biological diversity in Rondônia, as a source of information to establish better strategies for conservation and landscape management. In this study we present a checklist of Lecythidaceae in the region of the upper Madeira River and contribute improved information on species diversity of this important group of Amazonian plants, aiming at filling gaps in the floristic knowledge of Rondônia and the southern Amazon.

\section{Materials and methods}

The study area is located in the upper Madeira River region, in the municipality of Porto Velho, in southwestern Amazonia. The climate according to the Köppen classification is tropical humid (Awi type) with a relatively short dry season from June to August. The annual average temperature is $25.6^{\circ} \mathrm{C}$ and annual rainfall is between 1700 and $2000 \mathrm{~mm}$ (INMET, 2014). The landscape in the study area is physiognomically heterogeneous, forming different vegetation types such as dense and open upland forests, flooded forests, campinaranas (open woodland), savannas, and transitional vegetation, with different levels of human disturbance (RADAMBRASIL, 1978; Perígolo et al., 2016).

We examined about 370 Lecythidaceae specimens deposited in CEN, INPA and RON, (abbreviations following Thiers, 2015, continuously updated), the herbaria with the most comprehensive holdings from the upper Madeira River. Moreover, an extensive floristic survey carried out from 2008-2013 in the study region by the field team of the Embrapa Genetic Resources and Biotechnology herbarium (CEN) resulted in a large number of botanical collections from the area affected by the Jirau hydroelectric dam. Finally, we also carried out four field expeditions between February 2014 and March 2015 that specifically targeted Lecythidaceae, totalling a sampling effort of 42 days and 33 specimens collected in the study area. The collected material was deposited in CEN, with duplicates sent to IAN, INPA, NY, RON, $\mathrm{RB}, \mathrm{UB}$ and UFAC. Identification of the material was based on dichotomous keys and species descriptions available for the family (Prance \& Mori, 1979; Mori \& Prance, 1990; Mori \& Lepsch-Cunha, 1995; Hopkins \& Mori, 1999; Mori et al., 2010). The material examined was also compared with specimens identified by Scott Mori (specialist in the Lecythidaceae) available online at the NY Virtual Herbarium (http:// sciweb.nybg.org/science2/VirtualHerbarium. asp). The names and authors were checked in the International Plant Name Index (http://www.ipni. org). Information on geographical distribution of species was collated from The Lecythidaceae Pages (Mori et al., 2010), which contains a virtual monograph of the family, also from the Brazilian Flora Checklist (Smith et al., 2015). A vetted collection was selected as the voucher for each species found in the study area.

\section{Results}

We found 37 species of Lecythidaceae in the Upper Rio Madeira region (Table 1; Figs. 1, 2), belonging to seven genera: Eschweilera (21 species), Couratari (6), Cariniana (3), Gustavia L. (3), Allantoma (2), Bertholletia (1) and Lecythis (1). Data on stratum, habitat in the study area and geographical distribution and selected voucher specimens are provided in Table 1. Habit ranged from understory shrubs and treelets in Gustavia to emergent trees such as Bertholletia excelsa and Cariniana micrantha (Fig. 1I). The family grows in a wide range of habitats (Table 1), but most taxa were recorded in non-flooded forests (terra firme), while only four species were restricted to flooded forests (Couratari tenuicarpa, Eschweilera albiflora, E. ovalifolia and Gustavia augusta). Among the species recorded, nine are endemic to Brazil (Cariniana rubra, $C$. penduliflora, Couratari tauari, Eschweilera amazonica, E. atropetiolata, E. cyathiformis, E. pseudodecolorans, E. truncata and Lecythis serrata), while 13 species are new records of Lecythidaceae for Rondônia and one represents a possible new species (Table 1). 
TABLE 1. Species of Lecythidaceae found in the upper Madeira River in Rondônia ${ }^{a}$

\begin{tabular}{|c|c|c|c|c|}
\hline Species & Stratum & Habitat & $\begin{array}{l}\text { Global distribution / } \\
\text { Brazilian States }\end{array}$ & Voucher \\
\hline $\begin{array}{l}\text { Allantoma decandra (Ducke) } \\
\text { S.A.Mori, Ya Y.Huang \& Prance }\end{array}$ & em & tf & Bra, Col, Per / AC, AM, RO & $\begin{array}{l}\text { Catenacci } 70 \text { (CEN, INPA, NY, } \\
\text { RON, UB); Pereira-Silva } \\
1515610610 \text { (CEN, INPA, NY, } \\
\text { RON) }\end{array}$ \\
\hline $\begin{array}{l}\text { Allantoma pluriflora } \\
\text { S.A.Mori, Ya Y.Huang \& } \\
\text { Prance }\end{array}$ & $\mathrm{cp}$ & tf & Bol, Bra, Col, Per, Ven / AM, RO & $\begin{array}{l}\text { Catenacci } 74 \text { (CEN, INPA, NY, } \\
\text { RON, UB); Catenacci } 75 \text { (CEN, } \\
\text { INPA, NY, RON, UB) }\end{array}$ \\
\hline Bertholletia excelsa Bonpl. & $\mathrm{cp}, \mathrm{em}$ & $\mathrm{tf}, \mathrm{tfd}$ & $\begin{array}{l}\text { Bol, Bra, Col, GFr, Guy, Per, Sur, } \\
\text { Ven, TTb / AC, AM, AP, MT, PA, } \\
\text { RO, RR }\end{array}$ & $\begin{array}{l}\text { Catenacci } 58 \text { (CEN, INPA, NY, } \\
\text { RON); Pereira-Silva } 15638 \text { (CEN, } \\
\text { INPA, NY, RON) }\end{array}$ \\
\hline Cariniana micrantha Ducke & em & tf & Bol, Bra, Per / AC, AM, RO & $\begin{array}{l}\text { Catenacci } 57 \text { (CEN, INPA, NY, } \\
\text { RON, UB); Pereira-Silva } 16133 \\
\text { (CEN, INPA, NY, RON) }\end{array}$ \\
\hline Cariniana penduliflora Prance & ud & $\mathrm{tf}$ & $\mathrm{Bra} / \mathrm{RO}$ & Prance 8811 (INPA) \\
\hline Cariniana rubra Miers & ud & gal, sw & Bra / GO, MT, PA, RO, TO & $\begin{array}{l}\text { Catenacci } 48 \text { (CEN, INPA, NY, } \\
\text { RON); Catenacci } 71 \text { (CEN, INPA, } \\
\text { NY, UB, RON) }\end{array}$ \\
\hline Couratari atrovinosa Prance & $\mathrm{cp}$ & tf & Bra, Per / AM, RO & $\begin{array}{l}\text { Cid Ferreira } 8816 \text { (INPA); Nee } \\
34842 \text { (INPA) }\end{array}$ \\
\hline Couratari guianensis Aubl. & $\mathrm{cp}$ & tf & $\begin{array}{l}\text { Bra, Col, Cos, Ecu, GFr, Guy, Pan, } \\
\text { Sur, Ven / AC, AM, AP, MA, PA, RO }\end{array}$ & $\begin{array}{l}\text { Catenacci } 40 \text { (CEN, INPA, NY, } \\
\text { RON); Nelson P21262 (INPA) }\end{array}$ \\
\hline $\begin{array}{l}\text { Couratari macrosperma } \\
\text { A.C.Sm. }\end{array}$ & $\mathrm{cp}, \mathrm{em}$ & $\mathrm{tf}, \mathrm{tfd}$ & $\begin{array}{l}\text { Bol, Bra, Col, Per / AC, AM, BA, } \\
\text { ES, MT, PA, RJ, RO }\end{array}$ & $\begin{array}{l}\text { Catenacci } 52 \text { (CEN); } \\
\text { Pereira-Silva } 16340 \text { (CEN, INPA, } \\
\text { NY, RON) }\end{array}$ \\
\hline $\begin{array}{l}\text { Couratari multiflora (Sm.) } \\
\text { Eyma }\end{array}$ & ud & tf & $\begin{array}{l}\text { GFr, Guy, Sur, Ven / AM, AP, MA, } \\
\text { MT, PA, RO, RR }\end{array}$ & $\begin{array}{l}\text { Cid Ferreira } 8845 \text { (INPA); Lima } \\
69 \text { (RON) }\end{array}$ \\
\hline Couratari tauari O.Berg & $\mathrm{cp}$ & $\mathrm{tf}$ & $\mathrm{Bra} / \mathrm{AM}, \mathrm{PA}, \mathrm{RO}$ & $\begin{array}{l}\text { Catenacci } 64 \text { (CEN); Melo } 663 \\
\text { (CEN, INPA) }\end{array}$ \\
\hline Couratari tenuicarpa A.C.Sm. & $\mathrm{cp}, \mathrm{em}$ & $\begin{array}{l}\text { ig, } \\
\text { cam, va }\end{array}$ & Bra, Sur, Ven / AM, PA, RO & $\begin{array}{l}\text { Simon } 1494 \text { (CEN, INPA, NY, } \\
\text { RON); Simon } 2620 \text { (CEN, INPA, } \\
\text { NY, RON) }\end{array}$ \\
\hline Eschweilera albiflora Miers & cp, ud & ig & $\begin{array}{l}\text { Bol, Bra, Col, Per / AC, AM, MT, } \\
\text { PA, RO }\end{array}$ & $\begin{array}{l}\text { Pereira } 186(\mathrm{RON}) \\
\text { Pereira-Silva } 15663 \text { (CEN, INPA, } \\
\text { NY, RON) }\end{array}$ \\
\hline $\begin{array}{l}\text { Eschweilera amazonica } \\
\text { R.Knuth }\end{array}$ & $\mathrm{cp}, \mathrm{ud}$ & $\mathrm{tf}, \mathrm{tfd}$ & $\mathrm{Bra} / \mathrm{AM}, \mathrm{AP}, \mathrm{PA}, \mathrm{RO}, \mathrm{RR}$ & $\begin{array}{l}\text { Thomas } 5019 \text { (INPA); Thomas } \\
5057 \text { (INPA) }\end{array}$ \\
\hline $\begin{array}{l}\text { Eschweilera atropetiolata } \\
\text { S.A.Mori }\end{array}$ & $\mathrm{cp}$ & $\mathrm{tf}, \mathrm{tfd}$ & $\mathrm{Bra} / \mathrm{AM}, \mathrm{RO}$ & $\begin{array}{l}\text { Catenacci } 73 \text { (CEN, INPA, NY, } \\
\text { RON, UB); Thomas } 5109 \text { (INPA) }\end{array}$ \\
\hline Eschweilera bracteosa Miers & ud & $\mathrm{tf}$ & Bra, Col, Ecu, Per, Ven / AM, RO & $\begin{array}{l}\text { Mota } 99 \text { (INPA); Silveira } 111 \\
\text { (RON) }\end{array}$ \\
\hline $\begin{array}{l}\text { Eschweilera chartaceifolia } \\
\text { S.A.Mori }\end{array}$ & $\mathrm{cp}, \mathrm{ud}$ & $\begin{array}{l}\text { ig, tf, } \\
\text { tfd }\end{array}$ & Bra, Col, GFr, Guy, Per / AM, RO & $\begin{array}{l}\text { Nee } 34844 \text { (INPA); Simon } 1431 \\
\text { (CEN, INPA, NY, RON) }\end{array}$ \\
\hline $\begin{array}{l}\text { Eschweilera coriacea (DC.) } \\
\text { S.A.Mori }\end{array}$ & $\mathrm{cp}, \mathrm{ud}$ & tf, va & $\begin{array}{l}\text { Bol, Bra, Col, Ecu, GFr, Guy, Hon, } \\
\text { Pan, Per, Sur, Ven / AC, AM, AP, } \\
\text { MA, MT, PA, RO, RR }\end{array}$ & $\begin{array}{l}\text { Pereira-Silva } 15708 \text { (CEN); } \\
\text { Simon } 1675 \text { (CEN, INPA, NY, } \\
\text { RON) }\end{array}$ \\
\hline $\begin{array}{l}\text { Eschweilera cyathiformis } \\
\text { S.A.Mori }\end{array}$ & $\mathrm{cp}$ & $\mathrm{tf}$ & $\mathrm{Bra} / \mathrm{AM}$ & $\begin{array}{l}\text { Catenacci } 46 \text { (CEN, INPA, NY, } \\
\text { RON) }\end{array}$ \\
\hline $\begin{array}{l}\text { Eschweilera decolorans } \\
\text { Sandwith }\end{array}$ & $\mathrm{cp}, \mathrm{em}$ & tf & $\begin{array}{l}\text { Bra, Ecu, Guy, GFr, Per, Sur, Ven / } \\
\text { AM, RO, RR }\end{array}$ & $\begin{array}{l}\text { Simon } 1226 \text { (CEN, INPA, NY, } \\
\text { RON); Amaral-Santos } 3599 \text { (CEN, } \\
\text { INPA, NY, RON) }\end{array}$ \\
\hline $\begin{array}{l}\text { Eschweilera laevicarpa } \\
\text { S.A.Mori }\end{array}$ & $\mathrm{cp}, \mathrm{ud}$ & ig, tf & $\begin{array}{l}\text { Bra, Col, Ecu, GFr, Per, Ven / AM, } \\
\text { MT, RO, RR }\end{array}$ & $\begin{array}{l}\text { Catenacci } 55 \text { (CEN); } \\
\text { Pereira-Silva } 15462 \text { (CEN, INPA, } \\
\text { NY, RON) }\end{array}$ \\
\hline Eschweilera micrantha Miers & ud & tf & $\begin{array}{l}\text { Bra, GFr, Guy, Per, Sur, Ven / AM, } \\
\text { AP, MA, PA, RO }\end{array}$ & Mattos 68 (INPA) \\
\hline
\end{tabular}


TABLE 1. Continued

\begin{tabular}{|c|c|c|c|c|}
\hline Species & Stratum & Habitat & $\begin{array}{c}\text { Global distribution / } \\
\text { Brazilian States } \\
\end{array}$ & Voucher \\
\hline Eschweilera ovalifolia Nied. & $\mathrm{cp}, \mathrm{ud}$ & va & $\begin{array}{l}\text { Bol, Bra, Col, Ecu, Per / AC, AM, } \\
\text { RO, RR }\end{array}$ & $\begin{array}{l}\text { Catenacci } 59 \text { (CEN); Goulding } 6 \\
\text { (INPA) }\end{array}$ \\
\hline Eschweilera parviflora Miers & ud & tf & $\begin{array}{l}\text { Bol, Bra, GFr, Guy, Per, Ven / AM, } \\
\text { AP, RO }\end{array}$ & Vieira 259 (INPA) \\
\hline $\begin{array}{l}\text { Eschweilera parvifolia Mart. } \\
\text { ex DC. }\end{array}$ & $\mathrm{cp}$, ud & tf, va & $\begin{array}{l}\text { Bol, Bra, Col, Ecu, Guy, Per, Sur, } \\
\text { Ven / AC, AM, MT, RO, RR }\end{array}$ & $\begin{array}{l}\text { Pereira-Silva } 16232 \text { (CEN, INPA, } \\
\text { NY, RON); Pereira-Silva } 16316 \\
\text { (CEN, INPA, NY, RON) }\end{array}$ \\
\hline $\begin{array}{l}\text { Eschweilera pedicellata } \\
\text { (Rich.) S.A.Mori }\end{array}$ & $\mathrm{cp}$, ud & $\begin{array}{l}\text { ig, tf, } \\
\text { tfd }\end{array}$ & $\begin{array}{l}\text { Bra, GFr, Guy, Sur, Ven / AC, } \\
\text { AM, AP, PA, RO, RR }\end{array}$ & $\begin{array}{l}\text { Perigolo } 277 \text { (CEN, INPA, NY, } \\
\text { RON); Simon } 1826 \text { (CEN, INPA, } \\
\text { NY, RON) }\end{array}$ \\
\hline $\begin{array}{l}\text { Eschweilera } \\
\text { pseudodecolorans S.A.Mori }\end{array}$ & $\mathrm{cp}, \mathrm{em}$ & tf & $\mathrm{Bra} / \mathrm{AM}, \mathrm{RO}$ & $\begin{array}{l}\text { Catenacci } 56 \text { (CEN, INPA, NY, } \\
\text { RON); Pereira } 369 \text { (RON) }\end{array}$ \\
\hline $\begin{array}{l}\text { Eschweilera subglandulosa } \\
\text { Miers }\end{array}$ & $\mathrm{cp}$ & tf & $\begin{array}{l}\text { Bra, Guy, GFr, Per, Sur, Ven, } \\
\text { TTb / AM, RO }\end{array}$ & $\begin{array}{l}\text { Amaral } 2053 \text { (CEN, INPA, } \\
\text { NY, RON) }\end{array}$ \\
\hline $\begin{array}{l}\text { Eschweilera tessmannii } \\
\text { R.Knuth }\end{array}$ & ud & tf & Bra, Col, Ecu, Per/ AC, AM, RO & $\begin{array}{l}\text { Catenacci } 61 \text { (CEN, INPA, NY, } \\
\text { RON); Pereira-Silva } 15599 \text { (CEN, } \\
\text { INPA, NY, RON) }\end{array}$ \\
\hline Eschweilera truncata A.C.Sm. & $\mathrm{cp}$ & tf & $\mathrm{Bra} / \mathrm{AC}, \mathrm{AM}, \mathrm{RO}$ & $\begin{array}{l}\text { Catenacci } 50(\mathrm{CEN}) \text {; Simon } 1074 \\
\text { (CEN, INPA, NY, RON) }\end{array}$ \\
\hline $\begin{array}{l}\text { Eschweilera wachenheimii } \\
\text { (Benoist) Sandwith }\end{array}$ & ud & tf & Bra, Guy, GFr, Per, Sur / AM, RO & $\begin{array}{l}\text { Pereira-Silva } 15689 \text { (CEN, INPA, } \\
\text { NY, RON); Simon } 1025 \text { (CEN, } \\
\text { INPA, NY, RON) }\end{array}$ \\
\hline $\begin{array}{l}\text { Eschweilera aff. } \\
\quad \text { romeu-cardosoi S.A.Mori }\end{array}$ & $\mathrm{cp}$ & tf & - & $\begin{array}{l}\text { Catenacci } 63 \text { (CEN, INPA, NY, } \\
\text { RON, UB) }\end{array}$ \\
\hline Eschweilera sp. nov. & $\mathrm{cp}$ & tf & 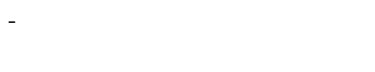 & $\begin{array}{l}\text { Pereira-Silva } 15647 \text { (CEN, INPA, } \\
\text { NY, RON) }\end{array}$ \\
\hline Gustavia augusta L. & ud & va & $\begin{array}{l}\text { Bol, Bra, Col, GFr, Guy, Per, Sur, } \\
\text { Ven / AC, AM, AP, BA, CE, GO, } \\
\text { MA, MT, PA, PB, PE, RO, RR }\end{array}$ & $\begin{array}{l}\text { Pereira-Silva } 15175 \text { (CEN, INPA, } \\
\text { NY, RON); } \\
\text { Pereira } 238 \text { (RON) }\end{array}$ \\
\hline Gustavia hexapetala $\mathrm{Sm}$. & $\mathrm{cp}$, ud & $\begin{array}{l}\text { tf, tfd, } \\
\text { va }\end{array}$ & $\begin{array}{l}\text { Bol, Bra, Col, Ecu, GFr, Guy, Per, } \\
\text { Sur, Ven / AC, AM, AP, MT, PA, } \\
\text { RO, RR }\end{array}$ & $\begin{array}{l}\text { Catenacci } 41 \text { (CEN, INPA, NY, } \\
\text { RON); Simon } 1860 \text { (CEN, INPA, } \\
\text { NY, RON) }\end{array}$ \\
\hline Gustavia poeppigiana O.Berg & ud & tf & $\begin{array}{l}\text { Bol, Bra, Col, Guy, Per, Ven / } \\
\text { AC, AM, PA, RO }\end{array}$ & $\begin{array}{l}\text { Catenacci } 54 \text { (CEN, INPA, NY, } \\
\text { RON); Perígolo } 195 \text { (CEN, INPA, } \\
\text { NY, RON) }\end{array}$ \\
\hline Lecythis serrata S.A.Mori & ud & tf & $\mathrm{Bra} / \mathrm{PA}, \mathrm{RO}$ & Equipe resgate 482 (RON) \\
\hline
\end{tabular}

${ }^{a}$ Species in bold are new records for Rondônia. Stratum: $\mathrm{cp}=$ canopy, em = emergent, ud = understory. Habitat: cam = campinarana, $\mathrm{gal}=$ gallery forest, $\mathrm{ig}=$ igapó forest, $\mathrm{tf}=$ terra firme forest, $\mathrm{tfd}=$ disturbed terra firme forest, va = várzea forest, $\mathrm{sw}=$ swamp (vereda). Countries: Bol = Bolivia, Bra = Brazil, Col = Colombia, Cos $=$ Costa Rica, Ecu $=$ Ecuador, GFr $=$ French Guiana, Guy = Guyana, Hon $=$ Honduras, Pan $=$ Panama, Per $=$ Peru, Sur $=$ Suriname, Ven $=$ Venezuela, $\mathrm{TTb}=$ Trinidad and Tobago. Brazilian states: $\mathrm{AC}=\mathrm{Acre}, \mathrm{AM}=$ Amazonas, $\mathrm{AP}=$ Amapá, $\mathrm{BA}=$ Bahia, $\mathrm{CE}=$ Ceará, $\mathrm{ES}=$ Espírito $\mathrm{Santo}, \mathrm{GO}=$ Goiás, $\mathrm{MA}=$ Maranhão, $\mathrm{MT}=$ Mato Grosso, $\mathrm{PA}=$ Pará, $\mathrm{PB}=$ Paraíba, $\mathrm{PE}=$ Pernambuco, $\mathrm{RJ}=$ Rio de Janeiro, $\mathrm{RO}=$ Rondônia, $\mathrm{RR}=$ Roraima, $\mathrm{TO}=$ Tocantins.

\section{Discussion}

According to the Brazilian Flora Checklist, Rondônia is the state with the third-richest Lecythidaceae flora $(28$ species, or 41 including the 13 new records reported here), behind Amazonas and Pará, with 79 and 43 species respectively (Smith et al., 2015). The current study represents a $46 \%$ increase in the known species richness of the family in Rondônia. Considering that this survey was restricted to the northern part of the state, our results suggest that the number of species of Lecythidaceae in Rondônia should increase as other regions and vegetation types are adequately surveyed. Botanical knowledge is lacking for most regions in the Brazilian Amazon, since collection effort is concentrated around major cities, rivers and highways. Not surprisingly, Brazil is the country with the largest collection gaps within Amazonia (Schulman et al., 2007). 

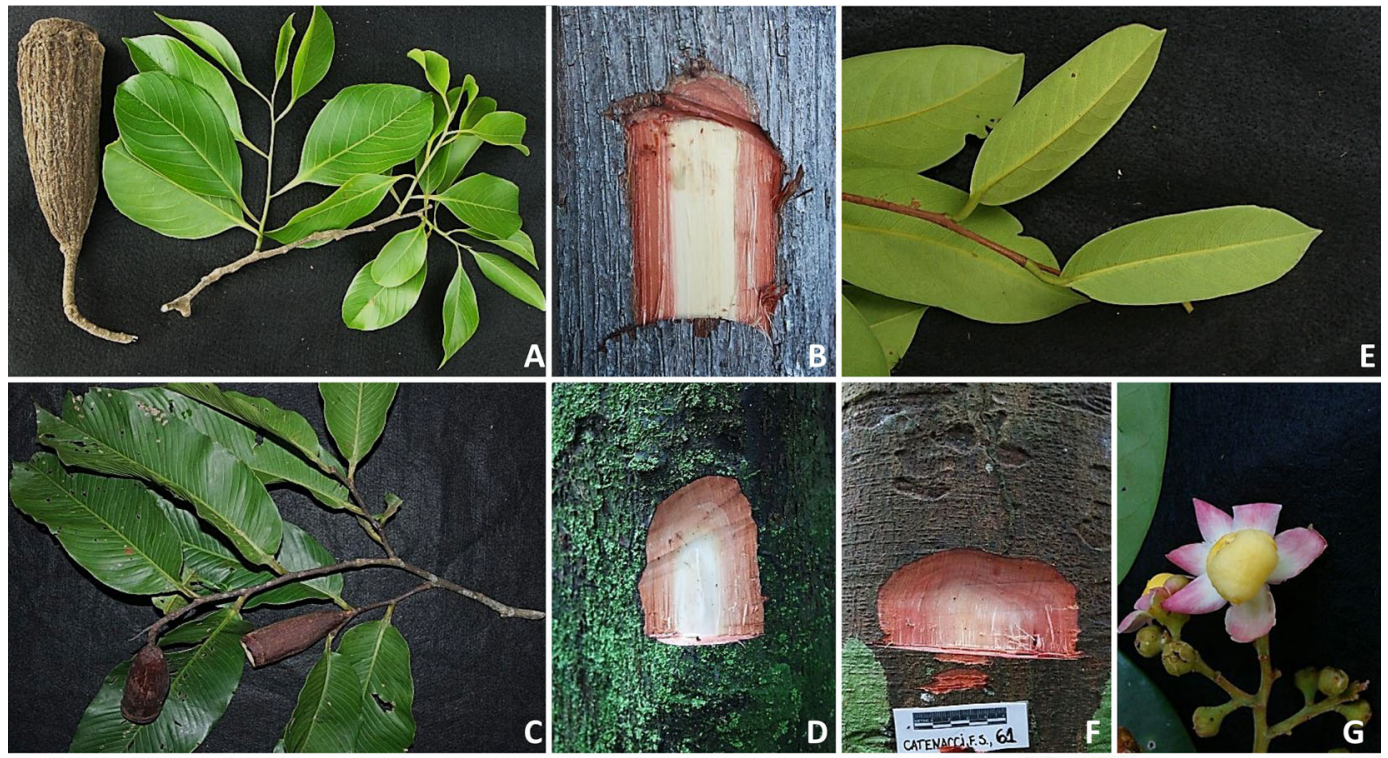

E
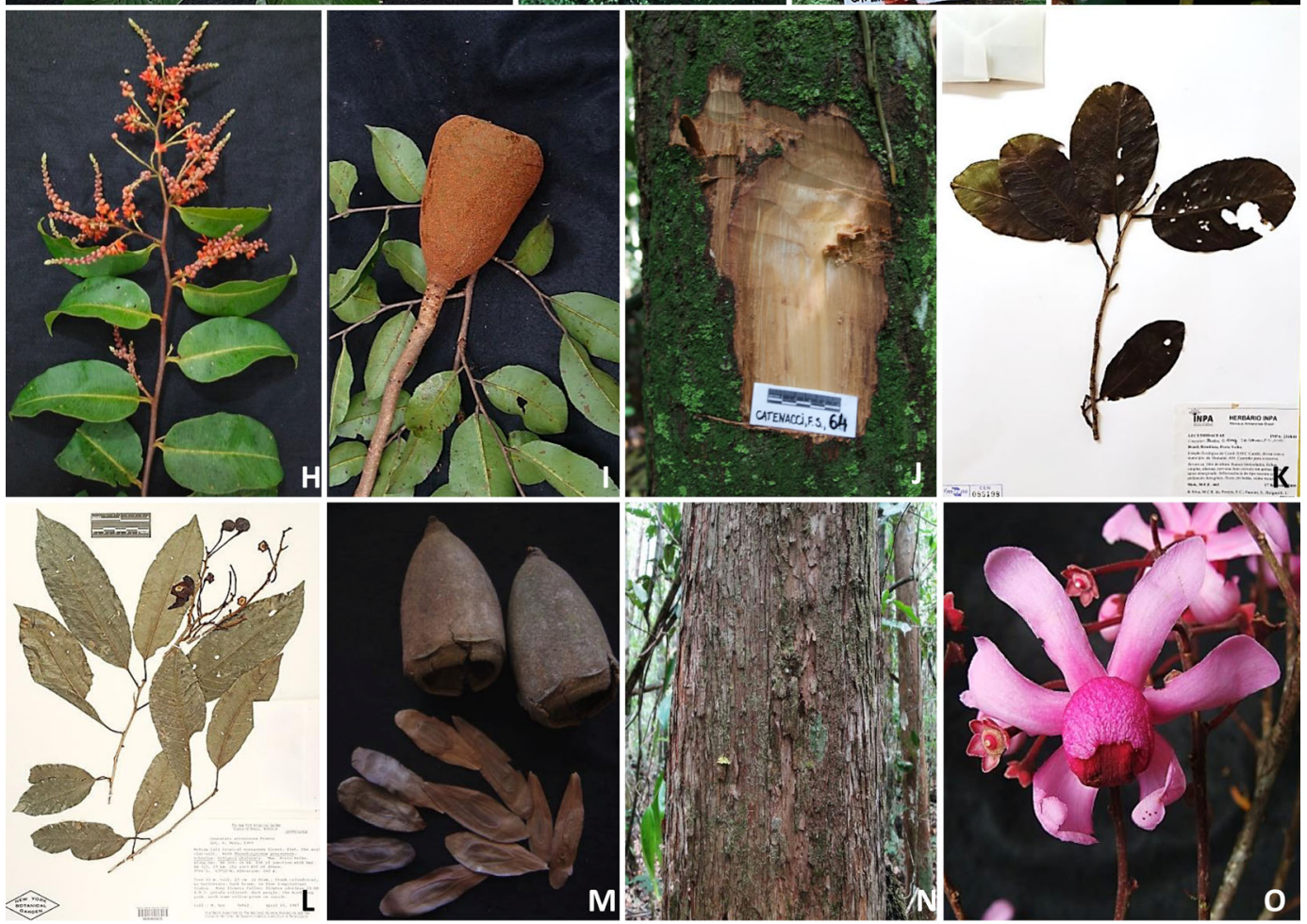

Fig. 1. Diversity of Lecythidaceae in the upper Madeira river, Rondônia. A-B. Allantoma decandra (Pereira-Silva 15610, Catenacci 70, respectively). C-D. Allantoma pluriflora (Catenacci 75, Catenacci 67). E-G. Eschweilera tessmannii (PereiraSilva 15730, Catenacci 61, Pereira-Silva 15599). H. Cariniana rubra (Catenacci 71). I. Cariniana micrantha (Pereira-Silva 16133). J-K. Couratari tauari (Catenacci 64, Melo 663). L. Couratari atrovinosa (Nee 34842). M. Couratari macrosperma (Pereira-Silva 16340). N-O. Couratari tenuicarpa (Simon 2620, Pereira-Silva 16223). The species group depicted in A-G is easily recognized in the field by orange inner bark. (Photos: A, E, G, I, M and O by G. Pereira-Silva; B-D, F, H, J-L by F. Catenacci; N by M. F. Simon). 

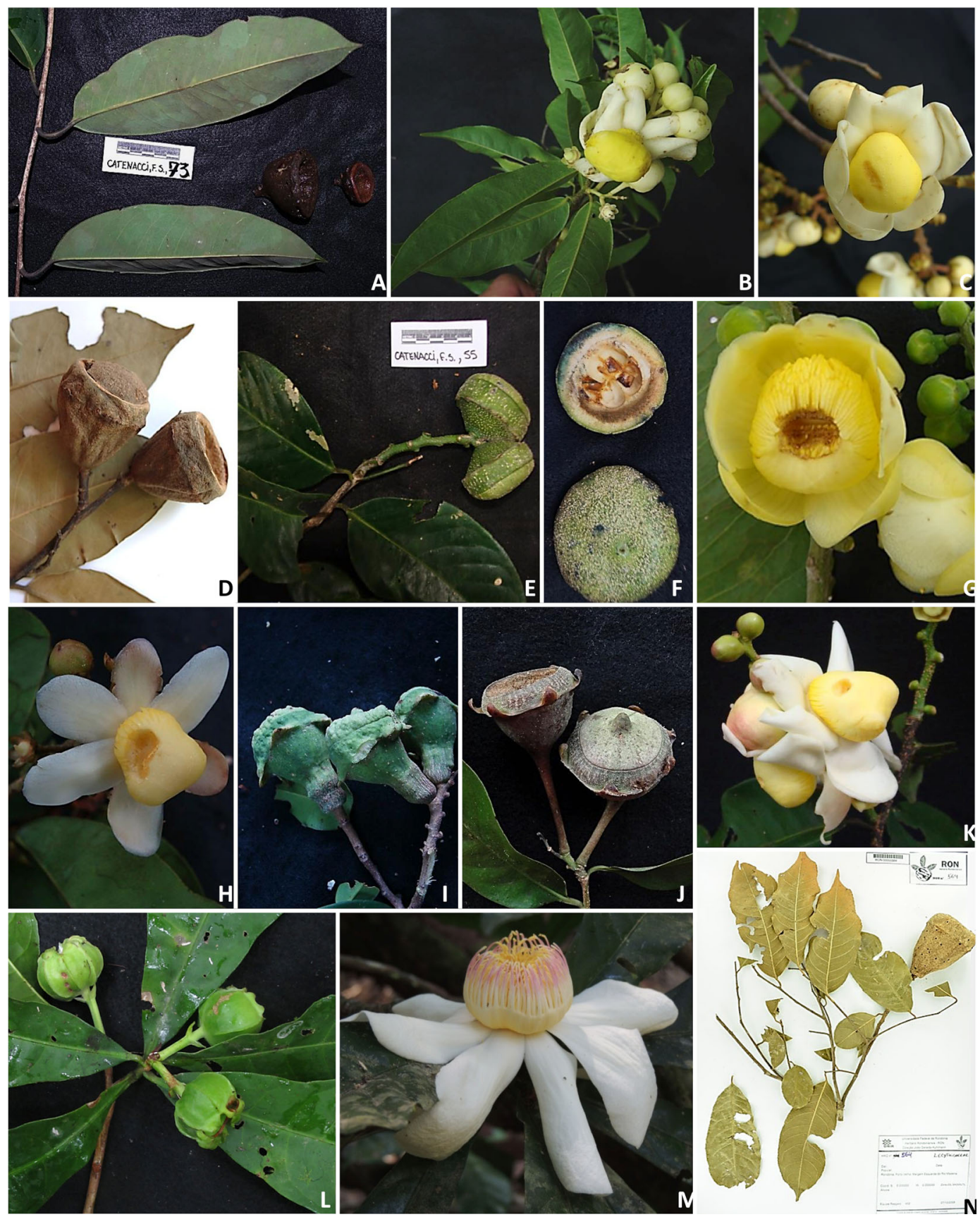

Fig. 2. Diversity of Lecythidaceae in the upper Madeira river, Rondônia. A. Eschweilera albiflora (Catenacci 73). B. Eschweilera chartaceifolia (Simon 1431). C. Eschweilera coriacea (Pereira-Silva 15708). D. Eschweilera cyathiformis (Catenacci 46). E-F. Eschweilera laevicarpa (Catenacci 55), showing bluish-green coloration of fruits when bruised. G. Eschweilera ovalifolia (Catenacci 59). H-I. Eschweilera parvifolia (Pereira-Silva 16232, 16316). J. Eschweilera pedicellata (Perígolo 277). K. Eschweilera pseudodecolorans (Catenacci 42). L. Gustavia hexapetala (Catenacci 41). M. Gustavia poeppigiana (Perígolo 195). N. Lecythis serrata (Equipe resgate 482). (Photos: A, D-G, K-L and N by F. Catenacci; B by M. F. Simon; C, H-I by G. Pereira-Silva; J and M by N. Perígolo). 
Several species found in the Upper Rio Madeira region are widely distributed in the Amazon region and adequately represented in herbaria (e.g., Bertholletia excelsa, Couratari guianensis, $C$. macrosperma, C. multiflora, Eschweilera albiflora, E. coriacea, E. micrantha, E. parvifolia, E. pedicellata, Gustavia augusta, G. hexapetala and G. poeppigiana), while others are endemic to Brazil but are also well-collected (Cariniana rubra, Eschweilera amazonica, E. atropetiolata, E. pseudodecolorans, E. truncata). However, four little-known species have drawn attention by the scarcity of records in herbaria (Cariniana penduliflora, Couratari atrovinosa, C. tauari and Lecythis serrata; Figs. 1 and 2), all with under 15 collections recorded in Brazil (speciesLink; www. splink.org.br). Cariniana penduliflora is known only from the type specimen collected in 1968 on the outskirts of Mutum-Paraná in Rondônia, a village flooded by the Jirau hydroelectric dam, while the other three species are known from few widely separated collections across the Amazon. Such scarcity of records suggests that these species may be globally rare, although this may also be an artifact of incomplete botanical knowledge in the region (Hopkins, 2007). Other poorly documented species are Allantoma pluriflora (Fig. 1C-D), which is relatively well collected in western Amazonia (Bolivia, Peru and Colombia) but has only a few collections in Brazil (western Amazonas state), and Eschweilera cyathiformis (Fig. 2D), which was previously known only from the Manaus region. The new records of these species for Rondônia could represent the extremes of their ranges but with gaps in between that could be filled by further collections.

Two taxa (Eschweilera sp. nov. and E. aff. romeo-cardosoi) could not be confidently assigned to any known species, but they are certainly distinct from other Lecythidaceae in the study area. One was identified as a possible new species by Lecythidaceae specialist S. Mori and requires additional plant material to permit a thorough description, since the flowers of this taxon are still not known. The other taxon has affinity with Eschweilera romeo-cardosoi, but its flowers and pedicels are much larger than those recorded for that species. This other possibly new taxon is morphologically intermediate between $E$. romeocardosoi and E. collina Eyma, with leaves and bark similar to the first and flowers and fruits similar to the latter. Although E. aff. romeocardosoi is a common species in some locations of the study area, more collections are needed to establish its identity.

Among the species found in the study area, five species are listed as threatened on the IUCN Red List (www.iucnredlist.org): Cariniana penduliflora (critically endangered), Couratari atrovinosa (endangered), Bertholletia excelsa, Couratari guianensis, and C. tauari (vulnerable). However, conservation assessments of these species must be reviewed considering recent occurrence data. According to the Red Book of Brazil's Flora (Martinelli \& Moraes, 2013), where B. excelsa is listed as vulnerable, Lecythidaceae is considered to be one of the ten botanical families of greatest interest for conservation and research because of restricted distributions or insufficient data for most species of the family. At a local scale, species of Lecythidaceae are threatened by both deforestation and infrastructure projects. Species restricted to the lowlands along the margins of the Madeira River are particularly affected by the construction of hydroelectric projects on the river, since most species of Lecythidaceae in the study are sensitive to flooding caused by dams.

Both herbarium and field work contributed to improved knowledge of the Lecythidaceae in the study area. Although recent field surveys added a substantial amount of botanical information, many of the new records presented here derived from specimens collected many years ago that had remained unidentified or misidentified in herbaria. For example, a search in the Brazilian network of herbaria (speciesLink) showed that $22 \%$ of the herbarium specimens of Lecythidaceae collected in Rondônia are determined only to genus, while about $30 \%$ of Eschweilera are unidentified to species. In addition, many Lecythidaceae collections are incomplete due to the difficulty of collecting large trees or are inappropriately dried or pressed, which causes the loss of floral features important for their identification. This situation, coupled with the great vegetative similarity between many of the species, makes identification of species of this family in the herbarium difficult (Mori \& Prance, 1987).

Lack of taxonomic expertise and unreliable identifications of herbarium specimens, together with geographically imbalanced collection effort, are major barriers to the improvement of our knowledge of the Amazon flora, making it difficult to accurately map species distributions and identify regions of endemism, key aspects of conservation planning (Hopkins, 2007). 
This situation is critical in the case of Rondônia, given the scenario of continued deforestation and habitat loss (Ferraz et al., 2005), including deforestation in protected areas (Verissimo et al., 2011) and the impacts of large hydroelectric projects (Fearnside, 2013). Rondônia should therefore be a priority area for floristic studies in the Amazon; it comprises a rich mosaic of landscapes in the transition between Cerrado and Amazonia, and it represents a probable center of plant endemism that is largely under-collected and has been facing high rates of deforestation for decades (Prance \& Campbell, 1988; Prance, 2001). Therefore, additional efforts are needed to inventory the state flora as a whole as well as in the protected areas, in order to evaluate the latter's effectiveness in conserving the landscape and floristic diversity of the state. Our results highlight a need for expanding floristic studies to areas in the Amazon that still lack adequate botanical information. Moreover, it is important to expedite the identification, mapping, and habitat analysis of the specimens generated by these studies. This would permit more robust conservation assessments and strategies for the Amazon flora.

\section{Acknowledgments}

We thank Glocimar Pereira-Silva, Antônio (Tonico) Barreto dos Santos, Valdeci (Dudu) Gomes, Natália Perígolo, Tamilis Rocha, Aécio Amaral-Santos and Marcelo Brilhante for their support during field expeditions; the curators of INPA and RON for loans of specimens; and Scott Mori for his encouragement and valuable advice on Lecythidaceae taxonomy. This research is part of first author's dissertation at University of Brasília) and she was supported by a scholarship from CAPES (Coordenação de Aperfeiçoamento de Pessoal de Nível Superior). This study is part of the project "Programa de Conservação da Flora na UHE Jirau" and was funded by Energia Sustentável do Brasil-ESBR.

\section{Literature cited}

APG II. 2003. An update of the Angiosperm Phylogeny Group classification for the orders and families of flowering plants: APG II. Botanical Journal of the Linnean Society 141: 399-436.
Fearnside, P. M. 2013. Decision-making on Amazon dams: Politics trumps uncertainty in the Madeira river sediments controversy. Water Alternatives 6: 313-325.

Ferraz, S. F. D. B., C. A. Vettorazzi, D. M. Theobald \& M. V. R. Ballester. 2005. Landscape dynamics of Amazonian deforestation between 1984 and 2002 in central Rondônia, Brazil: assessment and future scenarios. Forest Ecology Management 204: 69-85.

Hopkins, M. J. G. 2007. Modeling the known and unknown plant biodiversity of the Amazon Basin. Journal of Biogeography 34: 1400-1411.

Hopkins, M. J. G. \& S. A. Mori. 1999. Lecythidaceae. Pp. 273-287. In: J. E. L. S. Ribeiro, M. J. G. Hopkins, A. Vicentini, C. A. Sothers, M. A. S. Costa, J. M. Brito, M. A. D. Souza, L. H. P. Martins, L. G. Lohmann, P. A. C. L. Assunção, E. C. Pereira, C. F. Silva, M. R Mesquita \& L. C. Procópio (eds.), Flora da Reserva Ducke: Guia de identificação das plantas vasculares de uma floresta de terra-firme na Amazônia Central. Instituto Nacional de Pesquisas da Amazônia, Manaus, Brazil.

Huang, Y.Y., S. A. Mori \& L. M. Kelly. 2015. Toward a phylogenetic-based generic classification of neotropical Lecythidaceae-I. Status of Bertholletia, Corythophora, Eschweilera and Lecythis. Phytotaxa 203: 85-121.

INMET-Instituto Nacional de Meteorologia. 2014. Available at: http://www.inmet.gov.br/html/clima/mapas/. Accessed 20/Jan/2014.

Martinelli, G. \& M. A. Moraes (orgs.). 2013. Livro Vermelho da Flora do Brasil. Instituto de Pesquisas Jardim Botânico do Rio de Janeiro, Centro Nacional de Conservação da Flora, Rio de Janeiro, Brazil.

Mori, S. A. \& G. T. Prance. 1987. A guide to collecting Lecythidaceae. Annals of the Missouri Botanical Garden 74: 321-330.

- \& N. Lepsch-Cunha. 1995. The Lecythidaceae of a central Amazonian moist forest. Memoirs of the New York Botanical Garden 75: 1-55.

, N. P. Smith, Y. Y. Huang, G. T. Prance, L. M. Kelly \& C. C. Matos. 2015. Toward a phylogenetic-based generic classification of neotropical Lecythidaceae-II. Status of Allantoma, Cariniana, Couratari, Couroupita, Grias and Gustavia. Phytotaxa 203: 122-137.

, N. P. Smith, X. Cornejo \& G. T. Prance. 2010. The Lecythidaceae Pages (http://sweetgum.nybg.org/lp/index. php). The New York Botanical Garden, New York, USA

\& G. T. Prance. 1990. Lecythidaceae - Part II. The zygomorphic-flowered New World genera (Couroupita, Corythophora, Bertholletia, Couratari, Eschweilera and Lecythis). Flora Neotropica Monographs 21: 1-376.

C. C. Tsou, C. C. Wu, B. Cronholm \& A.

Anderberg. 2007. Evolution of Lecythidaceae with an emphasis on the circumscription of neotropical genera: Information from combined $n d h \mathrm{~F}$ and $t r n \mathrm{~L}-\mathrm{F}$ sequence data. American Journal of Botany 94: 289-301.

Moser, P., W. L. Oliveira, M. B. Medeiros, J. R. Pinto, P. V. Eisenlohr, I. L. Lima, G. P. Silva \& M. F. Simon. 2014. Tree species distribution along environmental gradients in an area affected by a hydroelectric dam in southern Amazonia. Biotropica 46: 367-376.

Perígolo N. A., M. B. Medeiros \& M. F. Simon. 2016. Vegetation types in the upper Maderia River in Rondônia. Brittonia (submitted).

Prance, G. T. 2001. Discovering the plant world. Taxon 50: 345-359. 
\& C. C. H. Jongkind. 2015. A revision of African Lecythidaceae. Kew Bulletin 70: 1-68.

\& E. K. Kartawinata. 2013. Lecythidaceae. Pp. 1118. In: H. P. Nooteboom \& P. C. van Welzen. (eds.), Flora Malesiana: Series I: Spermatophyta 21: 1-118. Naturalis Biodiversity Center, Leiden.

\& D. G. Campbell. 1988. The present state of tropical floristics. Taxon 37: 519-548.

\& S. A. Mori. 1979. Lecythidaceae - Part I. The actinomorphic-flowered New World Lecythidaceae (Asteranthos, Gustavia, Grias, Allantoma and Cariniana). Flora Neotropica Monographs 21: 1-270.

Procópio, L. C., M. Gayot, P. Sist \& I. D. Ferraz. 2010. As espécies de tauari (Lecythidaceae) em florestas de terra firme da Amazônia: Padrões de distribuição geográfica, abundâncias e implicações para a conservação. Acta Botanica Brasilica 24: 883-897.

RADAMBRASIL. 1978. Projeto Radambrasil. Vol. 18. Folha SC.20. Porto Velho; Geologia, geomorfologia, pedologia, vegetação e uso potencial da terra. Departamento Nacional de Produção Mineral, Rio de Janeiro, Brazil.

Schulman, L., T. Toivonen \& K. Ruokolainen. 2007. Analysing botanical collecting effort in Amazonia and correcting for it in species range estimation. Journal of Biogeography 34: 1388-1399.

Smith, N. P., S. A. Mori \& G. T. Prance. 2015. Lecythidaceae. In: Lista de Espécies da Flora do Brasil. Jardim Botânico do Rio de Janeiro. Avaiable at: http:// floradobrasil.jbrj.gov.br/jabot/floradobrasil/FB145. Accessed in: 26/Feb/2015.

ter Steege, H. et. al. 2013. Hyperdominance in the Amazonian tree flora. Science 342: 325-334.

Thiers, B. [continuously updated] 2015. Index Herbariorum: a global directory of public herbaria and associated staff. New York Botanical Garden's Virtual Herbarium. http:// sweetgum.nybg.org/science/ih/

Veríssimo, A., A. Rolla, M. Vedoveto \& S. M. de Furtada. 2011. Áreas protegidas na Amazônia Brasileira: Avanços e desafios. Imazon/ISA, Belém/São Paulo, Brazil. 